\title{
Telemonitoreo y control glucémico en pacientes con diabetes mellitus tipo 2
}

Telemonitoring and glycemic control in people with type 2 diabetes

Wild S.H, y col. PLoS Med 2016;13(7):e1002098.

\section{Objetivos}

Comparar la efectividad del telemonitoreo profesional sobre el autocontrol glucémico que realizan los pacientes con diabetes mellitus tipo 2 (DBT2).

\section{Diseño, lugar y pacientes}

Ensayo clínico aleatorizado con dos grupos paralelos, investigador-ciego, controlado, con aleatorización centralizada, realizado entre junio de 2011 y julio de 2013 en cuatro regiones del Reino Unido. Se incluyeron 321 pacientes con DBT2 y control glucémico deficiente, definido como hemoglobina glicosilada $(\mathrm{HbA1c})>58$ $\mathrm{mmol} / \mathrm{mol}(7,5 \%)$, que acuden a la consulta de médicos de familia.

\section{Intervención}

De los 321 pacientes, 160 fueron asignados al grupo de intervención y 161 al grupo control. En el grupo de intervención, el telemonitoreo implicó la auto-medición de la glucemia y la transmisión a un sitio web seguro dos veces por semana (glucemia en ayunas y otra no en ayunas), y medición de la presión arterial
(PA) y el peso por lo menos semanalmente, para la revisión de los parámetros por parte de los médicos de familia. El grupo control recibió atención habitual, con al menos una revisión anual.

\section{Medición de resultados principales}

El resultado primario fue la diferencia de medias ajustada de $\mathrm{HbA1c}$, y los resultados secundarios fueron las diferencias de medias ajustadas de presión arterial sistólica (PAS), la presión arterial diastólica (PAD) diurnas ambulatorias y el peso entre los grupos de tratamiento. El análisis fue realizado por intención de tratar.

\section{Resultados principales}

El resultado primario se describe en la tabla 1

En cuanto a los resultados secundarios, la PAS ambulatoria media ajustada fue $3,06 \mathrm{mmHg}$ menor (IC 95\% 0,56 a 5,56) y la PAD fue $2,17 \mathrm{mmHg}$ más baja (IC $95 \%$ 0,62 a 3,72), comparado con el grupo control. No hubo diferencias significativas en relación al peso, adherencia al tratamiento o calidad de vida.

Tabla 1. Efectividad del telemonitoreo profesional sobre el autocontrol glucémico.

\begin{tabular}{|c|c|c|c|c|c|c|}
\hline \multirow[t]{2}{*}{ Resultado } & \multicolumn{2}{|c|}{ Linea base } & \multicolumn{2}{|c|}{ Seguimiento } & \multirow{2}{*}{$\begin{array}{l}\text { Diferencia ajustada de } \\
\text { medias } \\
\text { (IC 95\%)** }\end{array}$} & \multirow[b]{2}{*}{ Valor } \\
\hline & $\begin{array}{l}\text { Grupo Telemonitoreo } \\
N=146 \text { Media (DE) }\end{array}$ & $\begin{array}{c}\text { Grupo Control } \\
N=139 \text { Media (DE) }\end{array}$ & $\begin{array}{c}\text { Grupo Telemonitoreo } \\
\mathrm{N}=146 \text { media (DE) }\end{array}$ & $\begin{array}{c}\text { Grupo Control } \\
N=139 \text { Media (DE) }\end{array}$ & & \\
\hline $\mathrm{HbA1c}(\mathrm{mmol} / \mathrm{mol})$ & 74,1 & $73(11,7)$ & $63,0(15,6)$ & $67,8(-14,7)$ & $-5,6(-8,81$ a $-2,38)$ & 0,007 \\
\hline HbA1c (\%) & $8,9(1,3)$ & $8,8(1,1)$ & $7,9(1.4)$ & $8,4(1,3)$ & $-0,51(-0,81$ a $-0,22)$ & 0,007 \\
\hline
\end{tabular}

** Ajustado por edad mayor a 70 años, sexo, centro, número de medicamentos para la diabetes, número de medicamentos para la hipertensión arterial, y frecuencia de autocontrol de la glucosa en los pacientes del estudio.

\section{Conclusión}

El apoyo profesional a través de telemonitoreo en pacientes con DBT2 atendidos por médicos de familia, mostró mejoras clínicamente significativas en el control de la glucemia.
Fuente de financiamiento: Beca del Programa de Investigación Aplicada de la Oficina de Científicos en Jefe. LifeScan (proporcionó los glucómetros y las tiras reactivas, no tuvo ningún otro papel en el estudio. Conflicto de interés: AS es miembro del Consejo Editorial de PLOS Medicine.

\section{Comentario}

Alrededor de 206 millones de personas con diabetes mellitus han sido diagnosticadas en el mundo. Esto ha despertado interés en el desarrollo de enfoques eficaces para mejorar el monitoreo de esta enfermedad, principalmente para evitar las complicaciones que representan el $80 \%$ del costo total de esta patología.

Este trabajo encontró evidencia sólida que sugiere que el telemonitoreo podría mejorar el control de la glucemia en pacientes con DBT2 y control glucémico deficiente que acuden a consulta de médicos de familia. Se utilizó acertadamente un diseño experimental para la evaluación de esta intervención en salud. Sin embargo, este experimento tuvo problemas en el enmascaramiento de la asignación de los participantes a los grupos de intervención y control. Esto puede llevar a un incremento en las cointervenciones y correcciones adicionales a los esquemas de tratamiento que podría influir en los hallazgos de este estudio. Un punto a favor es que tanto los resultados primarios y secundarios se basaron en mediciones objetivas, recolección de muestras de sangre, mapeo de la presión arterial y la medición del peso, por lo que la falta de enmascaramiento en teoría no afectaría de manera importante el riesgo de sesgo.

\section{Conclusiones del comentador}

La telemonitorización ha demostrado experimentalmente ser una alternativa interesante para un mejor control de la glucemia en pacientes con DBT2 que acuden a la consulta de un médico de familia. También hay beneficios adicionales para el control de la presión arterial en esta población. Sin embargo, es necesario tomar en cuenta el rigor metodológico, las diferencias poblacionales, el tiempo de seguimiento, el sistema de telemonitoreo estudiado, los costos y desenlaces importantes no medidos como la satisfacción y calidad de vida de los pacientes, antes de utilizar las conclusiones de este trabajo en nuestro contexto clínico.

Daniel Simancas-Racines y Camilo Félix [ Ctro. de Investigación en Salud Pública y Epidemiología Clínica. Ctro. Colaborador Cochrane de Ecuador. Ctro. de Telemedicina y Bioinformática. Facultad de Cs. de la Salud Eugenio Espejo. Univ. Tecnológica Equinoccial. dsimancas@ute.edu.ec ]

Simancas-Racines D, Félix C. Telemonitoreo y control glicémico en pacientes con diabetes mellitus tipo 2. Evid Act Pract Ambul. $2017 ; 20(1): 12$. Comentado de: Wild SH, y col. Supported Telemonitoring and Glycemic Control in People with Type 2 Diabetes: The Telescot Diabetes Pragmatic Multicenter Ran-domized Controlled Trial. PLoS Med 13(7): e1002098. PMID: 27458809.

\section{Referencias}

1. Guariguata L, col. Global estimates of diabetes prevalence for 2013 and projections for 2035. Diabetes Res Clin Pract. 2014; 103(2):137-49. doi: 10.1016/j.diabres.2013.11.002 PMID: 24630390

2. Gibson PG, y col. Self-management education and regular practitioner review for adults with asthma. Cochrane Database Syst Rev. 2000 (2: ):CD001117. PMID: 10796600 3. Farmer AJ, y col. Meta-analysis of individual patient data in randomised trials of self monitoring of blood glucose in people with non-insulin treated type 2 diabetes. BMJ. 2012; 344:e486. doi: 10.1136/bmj.e486 PMID: 22371867

4.Pal K, y col. Computer-based diabetes self-management interventions for adults with type 2 diabetes mellitus. Cochrane Database Syst Rev. 2013 Mar 28;(3):CD008776. doi: 10.1002/14651858.CD008776.pub2. Review. PubMed PMID: 23543567.

5. Malanda UL, y col. Self-monitoring of blood glucose in patients with type 2 diabetes mellitus who are not using insulin. Cochrane Database Syst Rev. 2012 Jan 18;1:CD005060. doi:10.1002/14651858.CD005060.pub3. Review. PubMed PMID: 22258959. 